\title{
Faster parameterized algorithms for Minimum FILL-IN
}

\author{
Hans L. Bodlaender \\ Pinar Heggernes \\ Yngve Villanger
}

Technical Report UU-CS-2008-042

December 2008

Department of Information and Computing Sciences

Utrecht University, Utrecht, The Netherlands

www.cs.uu.nl 
ISSN: 0924-3275

Department of Information and Computing Sciences Utrecht University

P.O. Box 80.089 3508 TB Utrecht

The Netherlands 


\title{
Faster parameterized algorithms for Minimum FiLL-IN
}

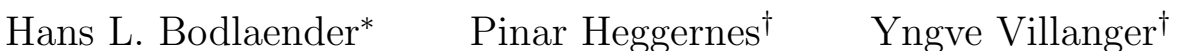

\begin{abstract}
We present two parameterized algorithms for the MINIMUM FILL-IN problem, also known as Chordal Completion: given an arbitrary graph $G$ and integer $k$, can we add at most $k$ edges to $G$ to obtain a chordal graph? Our first algorithm has running time $\mathcal{O}\left(k^{2} n m+3.0793^{k}\right)$, and requires polynomial space. This improves the base of the exponential part of the best known parameterized algorithm time for this problem so far. We are able to improve this running time even further, at the cost of more space. Our second algorithm has running time $\mathcal{O}\left(k^{2} n m+2.35965^{k}\right)$ and requires $\mathcal{O}^{*}\left(1.7549^{k}\right)$ space. To achieve these results, we present a new lemma describing the edges that can safely be added to achieve a chordal completion with the minimum number of edges, regardless of $k$.
\end{abstract}

\section{Introduction}

The Minimum FiLL-In problem asks, given as input an arbitrary graph $G$ and an integer $k$, whether a chordal graph can be obtained by adding at most $k$ new edges, called fill edges, to $G$. A chordal graph is a graph without induced cycles of length at least four. This is one of the most extensively studied problems in graph algorithms, as it has many practical applications in various areas of computer science. The problem initiated from the field of sparse matrix computations, where the result of Gaussian Elimination corresponds to a chordal graph, and minimizing the number of edges in a chordal completion is equivalent to minimizing the number of non-zero elements in Gaussian Elimination [23]. Among other application areas are data-base management systems [24], knowledge-based systems [18], and computer vision [6]. Since the problem was proved NP-complete [27], it has been attacked using various algorithmic techniques, and there exist polynomial-time approximation algorithms [20], exponential-time exact algorithms $[10,11]$, and parameterized algorithms $[16,5]$. The current best bounds are $\mathcal{O}^{*}\left(1.7549^{n}\right)$ time and space for an exact algorithm [11], and $\mathcal{O}\left((n+m) \frac{4^{k}}{k+1}\right)$ time for a parameterized algorithm [5], where $n$ and $m$ denote the number of vertices and edges of $G$, respectively, and the $\mathcal{O}^{*}$-notation suppresses factors polynomial in $n$.

In this paper we contribute with new parameterized algorithms to the solution of the Minimum FiLL-In problem. The field of parameterized algorithms, first formalized by Downey and Fellows [8], has been growing steadily and attracting more and more attention recently $[9,21]$. Informally, a parameterized algorithm computes an exact solution of the problem at

\footnotetext{
${ }^{*}$ Department of Information and Computing Sciences, Utrecht University, P.O. Box 80.089, 3508 TB Utrecht, the Netherlands. Email: hansb@cs.uu.nl

${ }^{\dagger}$ Department of Informatics, University of Bergen, PB 7803, 5020 Bergen, Norway. Email: \{pinar.heggernes, yngve.villanger\}@ii.uib.no Supported by the Research Council of Norway.
} 
hand, but the exponential part of the running time is limited to a (hopefully small) parameter, typically an integer. For the Minimum FilL-In problem, the natural parameter is $k$, the number of fill edges. The first parameterized algorithms for this problem were given by Kaplan et al. and appeared more than a decade ago $[16,17]$, with running times $\mathcal{O}\left(m 16^{k}\right)$ and $\mathcal{O}\left(k^{2} n m+k^{6} 16^{k}\right)$. A refined analysis of these algorithms by Cai gave the current best parameterized running time of $\mathcal{O}\left((n+m) \frac{4^{k}}{k+1}\right)[5]$.

We present two algorithms that improve on the basis of the exponential part of the running time of these parameterized algorithms. Central in our algorithms is a new result, describing edges that can always be added when computing a minimum solution. Based on this result, our first algorithm is intuitive and easy to understand, and requires $\mathcal{O}\left(k^{2} n m+3.0793^{k}\right)$ time and polynomial space. We are able to improve the base of the exponential part even further in a second algorithm, at the cost of more space. Our second algorithm, which is more involved, requires $\mathcal{O}\left(k^{2} n m+2.35965^{k}\right)$ time and $\mathcal{O}^{*}\left(1.7549^{k}\right)$ space.

This paper is organized as follows. In Section 2, we give some preliminary definitions and review well known results on chordal graphs and related notions. In Section 3, we give an important and useful result, where we characterize edges that can be safely added without disturbing optimality of a solution to the Minimum Fill In problem. Sections 4 and 5 give the descriptions and analyses of our algorithms, each of which will be presented in its own section: the algorithm that uses polynomial space is presented in Section 4, and Section 5 gives the algorithm that uses exponential space. For both of our algorithms, the input is an undirected graph $G=(V, E)$ and an integer $k$; each algorithm outputs either a minimum size set of at most $k$ fill edges, or NO if each chordal completion of $G$ requires at least $k+1$ fill edges. Finally, Section 6 gives some concluding remarks.

\section{Preliminaries}

All graphs in this work are undirected and simple. A graph is denoted by $G=(V, E)$, with vertex set $V$ and edge set $E(G)=E$. For a vertex subset $S \subseteq V$, the subgraph of $G$ induced by $S$ is $G[S]=(S,\{\{v, w\} \in E \mid v, w \in S\})$. The neighborhood of $S$ in $G$ is $N_{G}(S)=\{v \in(V \backslash S) \mid \exists w \in S:\{v, w\} \in E\}$. We write $N_{G}(v)=N_{G}(\{v\})$ for a single vertex $v$, and $N_{G}[S]=N_{G}(S) \cup S$. Subscripts are omitted when not necessary. A vertex $v$ is universal if $N(v)=V \backslash\{v\}$.

A vertex subset $S \subseteq V$ is a separator in $G$ if $G[V \backslash S]$ has at least two connected components. A connected component $C$ of $G[V \backslash S]$ is said to be associated to $S$, and it is called a full component if $N(C)=S$. Vertex set $S$ is a $u, v$-separator for $G$ if $u$ and $v$ are in different connected components of $G[V \backslash S]$, and a minimal $u, v$-separator for $G$ if no proper subset of $S$ is a $u, v$-separator. Two separators $S$ and $T$ are said to be crossing if $S$ is a $u, v$-separator for two vertices $u, v \in T$, in which case $T$ is an $x, y$-separator for two vertices $x, y$ in $S$ [22].

In general, $S$ is a minimal separator of $G$ if there exist $u$ and $v$ in $V$ such that $S$ is a minimal $u, v$-separator. If $S$ is a $u, v$ separator and $u$ is not in a full component associated to $S$, then $S$ is not a minimal $u, v$-separator, since $N(C) \subset S$ is also a $u, v$-separator, where $C$ is the component of $G[V \backslash S]$ that contains $u$. Hence the proposition below follows immediately.

Proposition 1 (Folklore) A set $S$ of vertices in a graph $G$ is a minimal u,v-separator if and only if $u$ and $v$ are in different full components associated to $S$. In particular, $S$ is a 
minimal separator if and only if there are at least two distinct full components associated to $S$.

A pair of vertices $\{u, v\}$ is a non-edge if $u$ and $v$ are not adjacent. For a vertex set $S$, we let $F(S)$ denote the set of non-edges in $G[S]$. $S$ is a clique if $F(S)=\emptyset$ or $|S|=1$. A clique is a maximal clique in $G$ if it is not a proper subset of another clique in $G$. A set of vertices that is both a clique and a separator is called a clique separator. A vertex $v$ is simplicial if $N(v)$ is a clique. A vertex set $U \subset V$ is a moplex if $G[U]$ is a clique, $N[v]=N[u]$ for any pair of vertices in $U$, and $N(U)$ is a minimal separator in $G$. Moplex $U$ is simplicial if $G[N(U)]$ is a clique.

A perfect elimination ordering (peo) of a graph $G=(V, E)$ is an ordering of the vertices of $G$ into $v_{1}, \ldots, v_{n}$, such that for each $i, 1 \leq i \leq n$, the higher indexed neighbors of $v_{i}$ form a clique, i.e., $v_{i}$ is a simplicial vertex in $G\left[\left\{v_{i}, v_{i+1}, \ldots, v_{n}\right\}\right]$.

A tree decomposition of a graph $G=(V, E)$ is a tree whose nodes correspond to subsets of $V$, called bags, that satisfies the following: every vertex of $V$ appears in a bag; for all $\{u, v\} \in E$, there is a bag where $u$ and $v$ appear together; for any vertex $u \in V$, the nodes of $T$ corresponding to the bags that contain $u$ induce a connected subtree of $T$. (We will simply use bags to denote both bags and nodes.) It follows that for two bags $X$ and $Y$ of a tree decomposition $T, X \cap Y$ is contained in every bag on the unique path between $X$ and $Y$ in $T$. A clique tree is a special kind of tree decomposition with a bijection between the nodes of the tree and the maximal cliques of $G$.

A chord in a cycle (path) is an edge that is between two non-consecutive vertices of the cycle (path). A graph is chordal, or triangulated, if every cycle on four or more vertices has a chord. A graph $H=(V, F)$ is a triangulation or chordal completion of a graph $G=(V, E)$ if $E \subseteq F$ and $H$ is chordal. The edges in $F \backslash E$ are called fill edges. $H$ is a minimal triangulation of $G$ if there is no triangulation $H^{\prime}=\left(V, F^{\prime}\right)$ of $G$ with $F^{\prime} \subset F$. A triangulation with the minimum number of edges is called a minimum triangulation. Every minimum triangulation is thus minimal. A set of vertices $S \subseteq V$ is a potential maximal clique (pmc) in $G$ if there is a minimal triangulation $H$ of $G$ where $S$ is a maximal clique in $H$.

For details on chordal graphs and triangulations, the reader can consult e.g., $[14,15]$. Here we give the minimum necessary background that is needed for our results and proofs.

Theorem $2([\mathbf{7}, \mathbf{1 2}, \mathbf{1 9}, \mathbf{4}, \mathbf{1 3}, \mathbf{2 6}])$ Let $G=(V, E)$ be a graph. The following are equivalent.

- $G$ is chordal.

- Every minimal separator of $G$ is a clique.

- Every minimal separator contained in the neighborhood of a vertex of $G$ is a clique.

- G has a peo.

- G has a clique tree.

Hence only chordal graphs have clique trees, whereas all graphs have tree decompositions (a trivial tree decomposition is one with a single bag containing all vertices of the graph). A clique tree is a very useful structure since it contains all the information of the minimal 
separators of a chordal graph. If we view each edge of a clique tree as the intersection of its endpoint maximal cliques, every edge of a clique tree of $G$ corresponds to a minimal separator of $G$, and every minimal separator of $G$ appears as an edge in every clique tree of $G$ [4]. It is also important to note that chordal graphs have at most $n$ maximal cliques [7], and hence at most $n-1$ minimal separators. It is an easy observation that every tree decomposition of an arbitrary graph $G$ corresponds to a triangulation $H$ of $G$ (not necessarily minimal) obtained by adding edges to $G$ so that every bag of the tree decomposition becomes a clique. The given tree decomposition of $G$ corresponds then to a clique tree of $H$.

The following characterization of minimal triangulations related to minimal separators is important for understanding our results.

Theorem 3 ([22]) Given an arbitrary graph $G$, a chordal graph $H$ is a minimal triangulation of $G$ if and only if $H$ is the result of making a maximal set of pairwise non-crossing minimal separators into cliques (by adding necessary edges to $G$ to achieve this).

From the above theorem it follows that if there is a clique separator $S$ in $G=(V, E)$, no minimal triangulation contains a fill edge between two vertices separated by $S$. Hence the Minimum FilL-In problem decomposes into subproblems $G[S \cup C]$ for each connected component $C$ of $G[V \backslash S]$. The same is true for minimal separators that are completed into cliques while computing a minimal triangulation [22].

In addition, we will use several times the following characterization of minimal triangulations related to moplexes.

Theorem 4 ([1]) Given an arbitrary graph $G$, a chordal graph $H$ is a minimal triangulation of $G$ if and only if $H$ is the result of repeatedly choosing a moplex $X$ and making the neighborhood of $X$ into a clique by adding the missing edges, before deleting $X$.

Finally, we end this section with the following easy but useful observation.

Proposition 5 (Folklore) Let $v_{1}, v_{2}, v_{3}, v_{4}, \ldots, v_{t}$ be a chordless cycle in a graph $G=(V, E)$, and let $H=(V, F)$ be a minimal triangulation of $G$, where $\left\{v_{1}, v_{3}\right\} \notin F$. Then there exists a fill edge $\left\{v_{2}, v\right\} \in F$ for some $v \in\left\{v_{4}, \ldots v_{t}\right\}$.

Proof. Since $v_{3}, v_{4}, \ldots, v_{t}, v_{1}$ is a path between $v_{1}$ and $v_{3}$ in $G$, there is also a path in $H$ between $v_{1}$ and $v_{3}$ involving only (a subset of) vertices $v_{3}, v_{4}, \ldots, v_{t}, v_{1}$. Let $v_{1}, u_{1}, u_{2}, \ldots, u_{r}, v_{3}$ be a shortest path of this kind between $v_{1}$ and $v_{3}$ in $H\left[V \backslash\left\{v_{2}\right\}\right]$. Since $v_{1}$ and $v_{3}$ are not adjacent, we can conclude that $r \geq 1$. Since $H$ is chordal and every induced subgraph of a chordal graph is also chordal, $H\left[\left\{v_{1}, u_{1}, \ldots, u_{r}, v_{3}, v_{2}\right\}\right]$ is chordal, has at least four vertices, and contains a cycle involving all its vertices. Since $H\left[\left\{v_{1}, u_{1}, \ldots, u_{r}, v_{3}\right\}\right]$ is a chordless path, $H\left[\left\{v_{1}, u_{1}, \ldots, u_{r}, v_{3}, v_{2}\right\}\right]$ contains fill edges and all fill edges are incident to $v_{2}$.

\section{Edges that can be safely added}

Before we start describing our algorithms, we present an important new result that describes fill edges that can be safely added when computing a minimum triangulation, independent of $k$. This is the first result of its kind to our knowledge, and it is crucial for our further results. 
Lemma 6 Given a graph $G=(V, E)$, let $S$ be a minimal separator of $G$ such that $|F(S)|=1$ and $S \subseteq N(u)$ for a vertex $u \in V$. Then there exists a minimum triangulation of $G$ that has the single element of $F(S)$ as a fill edge.

Proof. Let $H=(V, F), E \subset F$ be a minimum triangulation of $G$, and let $F(S)=\{\{x, y\}\}$. If $\{x, y\} \in F$, then there is nothing to prove, so assume that $\{x, y\} \notin F$. Let $T$ be a clique tree of $H$, and let $X$ and $Y$ be the closest pair of bags in $T$ such that $x \in X$ and $y \in Y$. Notice that $S \cup\{u\} \backslash\{x, y\}$ is a subset of every minimal $x, y$-separator, and by the above mentioned properties of tree decompositions, every vertex in $S \cup\{u\} \backslash\{x, y\}$ appears in every bag on the unique path from $X$ to $Y$ in $T$.

Let $C_{0}, C_{1}, \ldots, C_{r}$ be the connected components of $G[V \backslash S]$, let $u \in C_{0}$, and let $C_{0}, C_{1}, \ldots, C_{p}$ for $p \leq r$ be the connected components whose neighborhoods contain both $x$ and $y$. Clearly $H\left[C_{i} \cup S \cup\{u\}\right]$ is chordal for $i \in\{1, \ldots, r\}$, since any induced subgraph of a chordal graph is chordal.

We will now construct a tree decomposition $T^{\prime}$ of $G$ where the the bags that are not subsets of other bags will be exactly the maximal cliques of a triangulation $H^{\prime}=\left(V, F^{\prime}\right)$ of $G$, such that $\{x, y\} \in F^{\prime}$ and $\left|F^{\prime}\right| \leq|F|$. The first step is to make a bag $\mathcal{S}=S$. The expense of adding the edge $\{x, y\}$ will be compensated at a later point in the proof.

For $i \in\{p+1, \ldots, r\}$ take a clique tree $T_{i}$ of $H\left[N_{G}\left[C_{i}\right]\right]$ and add an edge from a maximal clique of $T_{i}$ containing $N_{G}\left(C_{i}\right)$ to $\mathcal{S}$. Notice that $N_{G}\left(C_{i}\right) \subset S$, and $N_{G}\left(C_{i}\right)$ does not contain both $x$ and $y$ and thus $H\left[N_{G}\left(C_{i}\right)\right]$ is a clique. We do not make any changes in subgraphs of this type.

For $i \in\{1, \ldots, p\}$, let $T_{i}$ be a clique tree of $H\left[N_{G}\left[C_{i}\right] \cup\{u\}\right]$, and let $X_{i}, Y_{i}$ be the closest pair of maximal cliques in $T_{i}$ containing $x$ and $y$, respectively. Notice that $u$ is contained in every maximal clique on the unique path from $X_{i}$ to $Y_{i}$ in $T_{i}$, and that $u$ has a fill edge to every vertex that appears in one of these maximal cliques, since $S$ separates $C_{i}$ from $C_{0}$ which contains $u$. Obtain the new tree $T_{i}^{\prime}$ by removing $u$ from $Y_{i}$, and replacing $u$ with $y$ in any other maximal clique of $T_{i}$. The number of fill edges will not increase, since all edges to $u$ from $C_{i}$ were fill edges, and these are now incident to $y$ instead. The edge $\{x, y\}$ is also added, but as mentioned this will be compensated for later. Now add an edge from $X_{i}$ in $T_{i}^{\prime}$ to $\mathcal{S}$.

By Proposition 1 the minimal separator $S$ has at least two full components, and thus $p \geq 1$, and $C_{0}$ and $C_{1}$ are two full components. When producing $T_{1}^{\prime}$ the vertex $u$ was replaced by $y$ in all maximal cliques of $T_{1}$ which contained $u$. Let $P_{1}=x, v_{1}, \ldots, v_{q}, y$ be a shortest path between $x$ and $y$ in $H\left[N_{G}\left[C_{1}\right]\right]$. Such a path must exist with $q \geq 1$ since $x$ and $y$ are not adjacent in $H$ and this subgraph is connected. Each vertex $v_{i}$ for $i \in\{1, \ldots, q\}$ is contained in some minimal $x, y$-separator of $H\left[N_{G}\left[C_{1}\right] \cup\{u\}\right]$, and is thus also contained in a maximal clique between $X_{1}$ and $Y_{1}$ by the previously mentioned properties of clique trees. Thus, it follows that $\left\{v_{1}, \ldots, v_{q}\right\} \subset N_{H}(u) \cap C_{1}$. We have now saved one edge since $\left\{u, v_{q}\right\}$ is a fill edge removed during the creation of $T^{\prime}$ and $\left\{v_{q}, y\right\}$ is already an edge of $H\left[N_{G}\left[C_{1}\right]\right]$. This edge is used to pay for the added edge $\{x, y\}$.

It remains to find a triangulation of $H\left[N_{G}\left[C_{0}\right]\right]$ containing $\{x, y\}$ as a fill edge. In [2] it is shown that if we have a chordal graph that does not contain an edge between two vertices $x$ and $y$, then the graph obtained by adding an edge between $x$ and $y$, and adding edges from $y$ to every vertex of every minimal $x, y$-separator is also chordal. Consequently, a chordal graph $H_{0}$ is obtained by adding edge $\{x, y\}$ and an edge from $y$ to every vertex of every minimal 
$x, y$-separator of $H\left[N_{G}\left[C_{0}\right]\right]$. Let $T_{0}$ be a clique tree of $H_{0}$, and let the final tree $T^{\prime}$ be obtained by adding an edge from $X_{0}$ in $T_{0}$ to $\mathcal{S}$. For an added fill edge $\left\{y, u^{\prime}\right\}$ where $u^{\prime}$ is contained in some minimal $x, y$-separator of $H\left[N_{G}\left[C_{0}\right]\right]$, there exists a fill edge $\left\{u^{\prime}, v_{i}\right\}$ that is removed from $H^{\prime}$, where $v_{i}$ is a vertex of the path $P_{1}$ in the other full component $C_{1}$. To see this, let $P_{0}=x, u_{1}, u_{2}, \ldots, u_{t}$ be a chordless path in $H\left[N_{G}\left[C_{0}\right]\right]$, such that $u_{j}=u^{\prime}$ for a $j \in\{1, \ldots, t\}$. This path exists, since $u^{\prime}$ is contained in a minimal $x, y$-separator of $H\left[N_{G}\left[C_{0}\right]\right]$. Consider again the clique tree $T$ of $H$, and let $X$ and $Y$ be the closest pair of bags in $T$ that contain $x$ and $y$, respectively. Every maximal clique on the unique path $X, Z_{1}, Z_{2}, \ldots, Z_{\ell}$ from $X$ to $Y$ in $T$ contains a vertex $v_{i}$ of path $P_{1}$ where $i \in\{1, \ldots, q\}$, and every vertex $u_{1}, \ldots, u_{t}$ of path $P_{0}$ is contained in some $Z_{i}$ where $i \in\{1, \ldots, \ell\}$. Thus, there exists a fill edge from $u^{\prime}$ to at least one vertex in $\left\{v_{1}, \ldots, v_{q}\right\}$ which is removed when obtaining the new tree $T^{\prime}$.

In total we have not added more edges than we have removed. For the final justification let us argue that $T^{\prime}$ is a tree decomposition of $G$. Every vertex is either contained in $S$ or one of the connected components of $G[V \backslash S]$ and is thus contained in one bag of $T^{\prime}$. For an edge $\{a, b\} \in E(G)$ we have the same, it is either between vertices in $S$ or between vertices in $G[N[C]]$ for some connected component $C$ of $G[V \backslash S]$. Finally, all bags containing a vertex $z$ induces a connected subtree of $T^{\prime}$, since this holds for every $T_{i}^{\prime}$ and for $T^{\prime}$ by the fact that a vertex is either completely in $T_{i}^{\prime}$ or the fact that every $T_{i}^{\prime}$ is directly attached to $\mathcal{S}$.

\section{An $\mathcal{O}^{*}\left(3.0793^{k}\right)$-time algorithm for Minimum FiLL-IN}

The first algorithm that we present uses polynomial time reductions and some branching rules. The input to the algorithm is an undirected graph $G=(V, E)$, and the algorithm either outputs a minimum size set of fill edges of size at most $k$, or NO if each triangulation of $G$ requires at least $k$ edges.

In the subproblems generated by this branching algorithm, some vertices have a marking. As will be clear when the subproblems are analyzed, sometimes we will have the choice of adding a set of fill edges or concluding with a set of vertices that each must be incident to a fill edge. These vertices will be marked, to give the desired restriction in the solution of resulting subproblems. More precisely, subproblems can be associated with problem instances of the form $(G, k, r, M)$ with $G=(V, E)$ a graph, $k$ and $r$ integers, and $M \subseteq V$ a set of marked vertices. For such an instance, we ask whether there exists a triangulation $H=(V, F)$ of $G$ with $|F \backslash E| \leq k$ and $2|F \backslash E|-|M| \leq r$, such that each vertex in $M$ is incident to a fill edge. We say that a vertex $v$ is marked if $v \in M$, and $r$ denotes the number of marks we still can place at later steps during the algorithm. From the original problem where $G$ and $k$ are given, the new initial problem instance is $(G, k, 2 k, \emptyset)$. Any triangulation of $G$ which requires $k$ fill edges is also a solution to the new instance since $r=2 k$ and $M=\emptyset$. Lemma 7 is a trivial consequence of our definition of subproblems.

Lemma 7 If $(G=(V, E), k, r, M)$ has a solution with $\{x, y\}$ as a fill edge, then $((V, E \cup$ $\{\{x, y\}\}), k-1, r-\gamma, M \backslash\{x, y\})$ has a solution, where $\gamma \in\{0,1,2\}$ is the number of unmarked endpoints of $\{x, y\}$.

At several points during our algorithm, we write: add an edge e to $F$ and update accordingly. Following Lemma 7, the update consists of decreasing $k$ by one, decreasing $r$ by the number of unmarked endpoints of $e$, and removing the marks of marked endpoints of $e$. If we 
add more edges, we do this iteratively. Whenever we mark a vertex, we decrease $r$ by one. Note that if two edges are added with a common endpoint, this endpoint is unmarked after the first addition, and thus causes a decrease of $r$ at the second addition. As an example consider the triangulation of a chordless cycle with five vertices. There exist two fill edges and thus four endpoints, but these two fill edges are incident to only three vertices altogether.

The algorithm is based on checking the existence of the structures described in the following paragraphs, and performing the corresponding actions. When a change is made to the input, we start again by checking trivial cases.

Trivial cases. First, the algorithm tests whether $G$ is chordal and $k \geq 0$ and $r \geq 0$. If so, it returns $\emptyset$. Next, it tests if $k \leq 0$ or $r \leq-1$. If so, it returns NO.

4-Cycles. Then, the algorithm branches on chordless cycles of length four (4-cycles). Suppose that $v, w, x, y$ induce a 4-cycle. Then, in any triangulation, $\{v, x\}$ is an edge or $\{w, y\}$ is an edge. The algorithm recursively solves the two subcases: one where we add $\{v, x\}$ as a fill edge and update accordingly, and one where we add $\{w, y\}$ as a fill edge and update accordingly.

An invariant of the algorithm is that each 4-cycle has at least two adjacent vertices that are not marked. Initially, this holds as all vertices are unmarked. Whenever we create a 4cycle by adding an edge, we unmark the endpoints of the added edge. Marks are only added in graphs that do not have a 4-cycle.

Note that we create in this case two subproblems. In each, $k$ is decreased by one, and $r$ is decreased by at least one. We will show by induction that the search tree formed by the algorithm has at most $a^{k} \cdot b^{r}$ leaves for inputs where we can use $k$ fill edges, and place at most $r$ marks. Thus, this case gives as condition $a^{k} \cdot b^{r} \geq 2 \cdot a^{k-1} \cdot b^{r-1}$, i.e, $a b \geq 2$.

Moplexes with marked and unmarked vertices. As a consequence of Theorem 4 and Proposition 5, we have the following result.

Lemma 8 Let $G$ be a graph and $U$ be a moplex in $G$. There is a minimum triangulation of $G$ that has a fill edge incident to each vertex in $U$, or there is a minimum triangulation of $G$ where $N(U)$ is a clique and no fill edge is incident to any vertex in $U$.

Proof. Note first that $N(U)$ is a minimal separator of $G$. By Theorem 3 and the subsequent discussion, $N(U)$ is turned into a clique in a minimal triangulation of $G$ if and only if no fill edge is added incident to a vertex of $U$. If $N(U)$ is not a clique in a minimal triangulation $H$ of $G$, then every vertex $u$ of $U$ is involved in a chordless cycle containing $u$, the endpoints of a missing edge $\{x, y\}$ in $N(U)$, and vertices of a shortest path between $x$ and $y$ in another full component associated to $N(U)$. Hence, by Proposition 5, every vertex of $U$ is incident to a fill edge in $H$.

Thus, when we have a moplex that contains marked and unmarked vertices, we mark all vertices of the moplex.

Finding moplexes with unmarked vertices. Then, the algorithm tests whether there is a moplex $U$ that contains no marked vertices. If there is no such moplex, the algorithm 
returns NO. Safeness of this step comes from the following lemma, which follows immediately from Theorem 4 by simply considering the first moplex that is removed after being made simplicial.

Lemma 9 Let $G$ be a graph and let $H$ be a minimum triangulation of $G$. There is a moplex $U$ in $G$, such that no fill edge is incident to any vertex of $U$ in $H$, and $N(U)$ is a clique in $H$.

We take such a moplex $U$, and let $S=N(U)$. We compute $F(S)$, i.e., the set of non-edges in the neighborhood of $U$.

Simplicial vertices. If $F(S)=\emptyset$, then all vertices in $U$ are simplicial. We recurse on the instance $(G \backslash U, k, r, M)$.

By Theorem 3 and the subsequent discussion, this instance is equivalent to, and a minimum set of fill edges for the new instance is also a minimum set of fill edges for, the original instance.

Moplexes missing one edge. Next, we test if $|F(S)|=1$. By Lemma 6 it is always safe to add the edge in $F(S)$, but in some cases we need to compensate for this by removing one mark from a vertex. The proof of the following Lemma relies heavily on Lemma 6 .

Lemma 10 Let $G=(V, E)$ be a graph and let $M \subseteq V$ be the set of marked vertices in $G$. Suppose there exists a minimum triangulation of $G$ such that each vertex in $M$ is incident to a fill edge. Let $u \in V$ be an unmarked vertex, and let $S \subseteq N(u)$ be a minimal separator with $F(S)=\{\{x, y\}\}$.

1. If there is a unique vertex $v^{*}$, such that $v^{*}$ is the last vertex on each chordless path from $x$ to $y$ through a full component of $S$ not containing $u$, then there is a minimum triangulation of $G$ that contains $\{x, y\}$ as a fill edge, and such that each vertex in $M \backslash\left\{v^{*}\right\}$ is incident to a fill edge.

2. If there is no unique vertex $v^{*}$, such that $v^{*}$ is the last vertex on each chordless path from $x$ to $y$ through a full component of $S$ not containing $u$, then there is a minimum triangulation of $G$ that contains $\{x, y\}$ as a fill edge, and such that each vertex in $M$ is incident to a fill edge.

Proof. Let $H=(V, F), E \subset F$, be a minimum triangulation of $G$, such that every vertex in $M$ is incident to at least one edge in $F \backslash E$. If $\{x, y\} \in F$, then there is nothing to prove, so let us assume that $\{x, y\} \notin F$. We will now analyze the triangulation $H^{\prime}=\left(V, F^{\prime}\right)$ constructed in the proof of Lemma 6 , and compare it to $H$. If every vertex in $M$ is incident to a fill edge in $F^{\prime} \backslash E$, then the proof of Lemma 6 can be applied directly.

Let us start by listing the vertices that in $H^{\prime}$ might loose their incident fill edges compared to $H$. For each connected component $C_{i}$, for $i \in\{1, \ldots, p\}$, fill edges incident to $u$ in $H\left[N_{G}\left[C_{i}\right] \cup\right.$ $\{u\}$ ] are moved to be incident to $y$ instead in $H^{\prime}$. This is unproblematic for any vertex not adjacent to $y$ in $G$, but for neighbours of $y$, this means that they loose an incident fill edge. We used this saved edge in the proof of Lemma 6 to pay for the new and added fill edge $\{x, y\}$, and thus there exists exactly one such saved edge, one such neighbor of $y$, and such component containing such a neighbor, since $H$ is an minimum triangulation. Let $\left\{v_{1}, \ldots, v_{q}\right\}$ 
be a chordless path from $x$ to $y$ in $H\left[N_{G}\left[C_{1}\right] \cup\{x, y\}\right]$. It follows that $v_{q}$ is the single vertex that has lost one incident fill edge in $H^{\prime}$ compared to $H$.. In the case of two different first vertices on a chordless path from $x$ to $y$ through $C_{1}$, two edges can be saved, which is a contradiction to $H$ being a minimum triangulation. Taking $v^{*}=v^{q}$ completes the proof. -

Suppose we have a moplex $U$, with $F(N(U))$ consisting of the single edge $\{x, y\}$. The condition of Lemma 10 now becomes: there is a vertex $v^{*}$ that is the last vertex on each chordless path in $G[V \backslash U]$ from $x$ to $y$. A simple modification of standard breadth first search allows us to find $v^{*}$ in linear time, if it exists. If $v^{*}$ exists, we remove its marking. Then, in both cases, we add the edge $\{x, y\}$ and update accordingly.

In this case, we possibly decrease $k$ by one, and increase $r$ by one. This gives us as condition on the running time constants: $a \geq b$.

The condition is not symmetric. We can apply the condition with roles of $x$ and $y$ switched and save a marking in some cases.

Branching on moplexes. If none of the earlier tests succeeds, we arrive at the last branching, performed on a moplex $U$ with all vertices in $U$ unmarked.

Recall Lemma 8. It dictates which two subproblems we consider. In the first subproblem, we mark all vertices in $U$. In the second subproblem, we add all edges in $F(N(U))$ and update accordingly. In the first, $r$ is decreased by $|U|$. In the second, $|F(N(U))| \geq 2$, as otherwise there is no pair of edges with a common endpoint in $F(N(U))$, so $k$ is decreased by two. Note that there must be a vertex that is common to two elements of $F(N(U))$ : if not, then suppose $\{x, y\}$ and $\{v, w\}$ are elements in $F(N(U))$, but no other combination of $x, y, v$, and $w$ is an element of $F(N(U))$. Then, these four vertices form a 4-cycle, which is a contradiction. Thus, in the second subproblem, $r$ is also decreased by at least one.

This gives us as condition for the running time analysis: $a^{k} \cdot b^{r} \geq a^{k-2} \cdot b^{r-1}+a^{k} \cdot b^{r-1}$, or $a^{2} b \geq 1+a^{2}$.

Each of these subproblems is solved recursively, and from these solutions, we then return the best one, adding $F(N(U))$ to the set returned by the second subproblem except when it returned NO.

Analyzing the running time. By standard graph algorithmic tools, each recursive call can be performed in time $\mathcal{O}(\mathrm{nm})$, except that the checking for all 4-cycles before any other operation is done requires $\mathcal{O}\left(\mathrm{m}^{2}\right)$ time, once. We now analyze the number of recursive calls in the search tree. We start with an instance with $r=2 k$, so the running time of the algorithm is bounded by $a^{k} \cdot b^{2 k}$. Each of the steps gave a condition on $a$ and $b$, and we get as minimum $a b^{2}=3.0793$ when we set $a=1.73205$ and $b=1.33334$. Thus, the total running time becomes $\mathcal{O}\left(m^{2}+n m \cdot 3.0793^{k}\right)$.

By the results of [17] and [20] it is possible to reduce a given instance $(G=(V, E), k)$ of Minimum Fill-In to an equivalent instance $\left(G^{\prime}=\left(V^{\prime}, E^{\prime}\right), k^{\prime}\right)$ where $k^{\prime} \leq k$ and $\left|V^{\prime}\right|=$ $\mathcal{O}\left(k^{2}\right)$, in $\mathcal{O}\left(k^{2} n m\right)$ time. By preprocessing the input by such an algorithm we get an additive time cost of $\mathcal{O}\left(k^{2} n m\right)$ but the size of $n$ and $m$ have been reduced to respectively $\mathcal{O}\left(k^{2}\right)$ and $\mathcal{O}\left(k^{4}\right)$. Thus, the time complexity for our algorithm becomes $\mathcal{O}\left(k^{2} n m+3.0793^{k}\right)$.

Theorem 11 The Minimum FiLL-In problem can be solved in $\mathcal{O}\left(k^{2} n m+3.0793^{k}\right)$ time, using polynomial space. 


\section{An $\mathcal{O}^{*}\left(2.35965^{k}\right)$-time algorithm for MINIMUM FILL-IN}

In this section, we give a second algorithm for the Minimum FILL-In problem. This algorithm uses less time as a function of $k$, at the cost of exponential space as a function of $k$. Like the previous algorithm, we create subinstances with some vertices marked and with an additional parameter $r$, which is the number of marks that still can be handed out.

An important difference to the algorithm of Section 4 is that the mark is a vertex set containing the vertices which are candidates to add a fill edge incident to. I.e., marks are annotated. This is to properly execute the steps where we partition on clique separators. The algorithm involves a more extensive analysis of subproblems, a mixing of eliminating moplexes with partitioning the graph on clique separators, resolution of cycles with four vertices, and a resolution of certain cases with the exact algorithm, recently given by Fomin and Villanger [11]. We also allow that the algorithm returns solutions that do not respect marks. When there is a solution respecting marks with $\alpha$ fill edges, the algorithm may return any solution with at most $\alpha$ fill edges. If the algorithm returns NO, we know there is no solution that respects marks. This is needed for the step where we use the exact algorithm by Fomin and Villanger [11], as we ignore the marks in that step.

With our algorithm description, we will also make the first steps towards the time analysis. We derive a number of conditions on a function $T(k, r)$, such that the running time of all recursive calls that originate at a node with parameters $k$ (number of fill edges) and $r$ (number of marks that still can be placed) is bounded by $T(k, r)$ times a function, polynomial in $n$, not depending on $k$. As the time for non-leaf nodes of the search tree is bounded by a polynomial in $n$ times the time for leaf nodes, we only count the time at leaf nodes. We want to show that $T(k, r) \leq a^{k} \cdot b^{k} \cdot o(k)$ and derive some conditions on $a$ and $b$.

The algorithm consists of carefully handling subproblems of various types. We describe in the next paragraphs which conditions are tested, in what order, and what steps are executed if a certain condition holds. First we will present some polynomial-time reduction rules. Several cases are similar to or the same as in our previous algorithm.

Trivial cases. Exactly like in the algorithm of Section 4, if $G$ is chordal and $k \geq 0$ and $r \geq 0$, then we return the empty set. If $G$ is not chordal, and $k \leq 0$ or $r \leq-1$, we return No.

Universal vertex. If $G$ contains a universal vertex then we simply remove this vertex. This is safe, since no chordless cycle of length at least 4 contains such a vertex.

Simplicial vertices. If an unmarked vertex is simplicial then we remove the vertex, and obtain an equivalent instance. If a marked vertex is simplicial then we return NO, since no minimal triangulation will add fill edges incident to a simplicial vertex by Theorem 3 .

Clique separators. Then, the algorithm tests if there is a clique separator. If there is a clique separator $S$, then let $V_{1}, \ldots, V_{r}$ be the vertex sets of the connected components of $G[V \backslash S]$. We create now $r$ subinstances, with graphs $G\left[S \cup V_{1}\right], \ldots, G\left[S \cup V_{r}\right]$.

Vertices in subinstances in $V \backslash S$ keep their marks. A marked vertex in $S$ is marked in only one subinstance, containing the annotated vertex set related to the mark. We will describe this more in detail when the annotated vertices are defined. 
These subproblems are now independent by Theorem 3 and its subsequent discussion. First, we test for each subproblem if there is a solution with at most two fill edges. If so, we solve this subproblem in polynomial time and use the obtained solution to reduce the parameter of the remaining problems.

When we have $\alpha$ subproblems each of whose solutions requires at least three fill edges, each can add at most $k-3 \alpha+3$ fill edges. For each of these subinstances is solved with parameter $k-3 \alpha+3$; we answer yes if the total of all minimum triangulation for all subinstances is at most $k$.

Thus, we need to choose $a$ and $b$ such that $T(k, r) \leq \alpha \cdot T(k-3 \alpha+3, r)$ for all $\alpha>1$. This gives $a^{3 \alpha-3} \geq \alpha$, which holds for every integer $\alpha>1$ and $a \geq 1.3$.

A minimal separator missing one edge. The next step is similar to the steps in our previous algorithm that use Lemma 10, but now we apply it also to vertices that do not belong to a moplex.

We test if there is an unmarked vertex $v$ and a minimal separator $S \subseteq N(v)$, such that $F(S)$ contains only one edge. If we have such a minimal separator $S$, we add the edges in $F(S)$, test if vertex $v^{*}$ described in Lemma 10 exists, if $v^{*}$ exists, we remove the mark of $v^{*}$, update accordingly, and solve recursively the remaining instance.

If this instance returns NO, we return NO, otherwise we return the union of $F(S)$ and the solution found by the instance. This again gives as condition for the running time analysis: $a \geq b$.

Above, we have given a number of reduction steps. We repeat applying reductions, until none of the above reduction steps applies, and then consider, in the given order, the following branching steps.

4-Cycles. Exactly like in the algorithm of Section 4, we now test if there is a chordless 4 -cycle, and branch on the two ways of adding an edge between non-adjacent vertices in the cycle. Again, we get as condition $a b \geq 2$.

Minimal separator $\boldsymbol{S}$ with $|\boldsymbol{F}(\boldsymbol{S})| \geq 3$. Next we test if there is an unmarked vertex $v$, and a minimal separator $S \subseteq N(v)$ with $|F(S)| \geq 3$. If so, we branch on this vertex, similarly as in the previous algorithm: we create two subinstances and recurse on each of these, and then output the smallest fill set of these instances, treating NO as a solution of size $\infty$.

In one subinstance, we add all fill edges in $F(S)$, and $k$ is decreased by $|F(S)|$. For each unmarked vertex incident to an edge of $F(S), r$ is decreased by one. For each vertex incident to $j>1$ edges in $F(S), r$ is decreased by $j-1$. We also remove all marks from vertices incident to edges in $F(S)$.

In the other subinstance, we mark vertex $v$, but we also have to define the annotation for the mark of $v$. Let $W$ be a connected component of $G[V \backslash N[v]]$ not containing $v$ such that $N(W)=S$. The connected component $W$ exists by definition of $S$. The annotated vertices for the mark of $v$ will be $W$. Let us justify this:

Since $S$ is not completed into a clique, there is an edge $\{x, y\} \in F(S)$ which is not used as a fill edge in the optimal solution we are searching for. Vertex set $W$ is a full component of $S$, and thus there exists a chordless path $u_{1}, u_{2}, \ldots, u_{r}$ from $x$ to $y$ only containing vertices of $W$. Vertex set $\left\{y, v, x, u_{1}, u_{2}, \ldots, u_{r}\right\}$ induces a cycle in $G$. By Proposition $5, v$ has a fill edge 
to one of the vertices in $\left\{u_{1}, u_{2}, \ldots, u_{r}\right\}$ if $\{x, y\}$ is not a fill edge. Since we do not know which one of the edges in $F(S)$ is not added when $v$ is marked, we use $W$ as the annotation and in this way ensure that the correct vertex is in the set.

Lemma 12 Given a graph $G$, let $S \subset V$ be a clique separator, let $v \in S$ be a marked vertex, and let $X$ be the annotation of $v$. Then there exists a connected component $W$ of $G[V \backslash S]$ such that $X \subseteq W$.

Proof. Note first that $S \cap X=\emptyset$, since no vertex of $X$ is adjacent to $v$ when $v$ is marked. Secondly, the mark of $v$ is removed when fill edge $\{x, v\}$, where $x \in X$, is added. Finally, $S$ does not separate any pair of vertices in $X$, since $S$ contains no vertices in $X$, and $G[X]$ is connected.

Again, we return the smallest solution found by the two subinstances, treating NO as a solution of size $\infty$.

Similar arguments as before show correctness of this step. In a minimum triangulation, we must either add all edges in $F(S)$ or vertex $v$ will be incident to a fill edge. In the first subinstance, we have $k$ reduced by $|F(S)| \geq 3$, and $r$ reduced by at least two. This can be seen as follows. There are no chordless 4-cycles in $G$, and thus the edges of $F(S)$ form a connected graph with vertex set $S$. A consequence of this is that at most $|F(S)|-1$ vertices will be incident to the edges in $F(S)$. When updating, $r$ is decreased by $2 \cdot|F(S)|$ minus the number of marked vertices that are endpoint of an edge in $F(S)$, which is at least two. In the second subinstance, $r$ is decreased by one, as we place one mark. This gives: $T(k, r) \leq T(k-3, r-2)+T(k, r-1)$, leading to the condition $a^{3} b^{2} \geq 1+a^{3} b$.

Notice that after this step has been carried out, for any remaining minimal separator $S$ contained in the neighborhood of an unmarked vertex, $|F(S)|=2$.

Split the problem into two non-chordal subproblems. Let $v$ be an unmarked vertex, let $S$ be a minimal separator in $N(v)$, and let $G^{\prime}$ be the resulting graph where the elements of $F(S)$ are added to $G$ as fill edges. We test if there are two connected components $W_{1}$ and $W_{2}$ of $G[V \backslash S]$ where $G^{\prime}\left[N\left[W_{1}\right]\right]$ and $G^{\prime}\left[N\left[W_{2}\right]\right]$ are non-chordal. This test can be carried out in polynomial time [24].

If this test fails for all unmarked $v$ and minimal separators in $N(v)$, we continue with the next step. Suppose now that we have found a vertex $v$ and two connected components $W_{1}$ and $W_{2}$ of $G[V \backslash S]$ with $G^{\prime}\left[N\left[W_{1}\right]\right]$ and $G^{\prime}\left[N\left[W_{2}\right]\right]$ non-chordal.

We will then know that at least one fill edge will be required for each of the connected components $W_{1}$ and $W_{2}$ in the case where $S$ is completed into a clique. The algorithm proceeds as follows: Check if one of the subproblems $G^{\prime}\left[N\left[W_{1}\right]\right]$ or $G^{\prime}\left[N\left[W_{2}\right]\right]$ can be triangulated by adding at most three fill edges. We can use any FPT-algorithm for Minimum FILL-IN to do this in polynomial time.

First, suppose this check holds. Without loss of generality, suppose $G^{\prime}\left[N\left[W_{1}\right]\right]$ can be triangulated by adding $k_{1} \leq 3$ fill edges. We recurse on two subinstances. The first subinstance handles the case where $S$ is completed into a clique. Here we need to recurse on $G^{\prime}\left[N\left[W_{2}\right]\right]$, where $k$ is reduced by $|F(S)|+k_{1} \geq 3$. Similar as above, the at least three fill edges form a connected graph on the set of its endpoints, and hence we save at least two marks. In the second subinstance, we mark $v$. Thus, in this case, we get the recursive condition: $T(k, r) \leq$ $T(k-3, r-2)+T(k, r-1)$, giving $a^{3} b^{2} \geq 1+a^{3} b$. 
Suppose neither $G^{\prime}\left[N\left[W_{1}\right]\right]$ nor $G^{\prime}\left[N\left[W_{2}\right]\right]$ can be triangulated by adding at most three fill edges. We consider again two subinstances, but directly split the first instance again. In the first main subinstance, we complete $S$ into a clique. Now, $S$ is a clique separator. Thus, we can instead solve two smaller subinstances, and recurse on $G^{\prime}\left[N\left[W_{1}\right]\right]$ and $G^{\prime}\left[V \backslash N\left[W_{1}\right]\right]$. In each of these subinstances, the parameter $k$ is reduced by six: we used two fill edges for completing $S$ and must use at least four fill edges in the other subinstance. As the two fill edges in $S$ share an endpoint, we decrease $r$ by one. In the second main subinstance, we mark $v$. This gives the recursive condition: $T(k, r) \leq 2 T(k-6, r-1)+T(k, r-1)$, giving $a^{6} b \geq 2+a^{6}$.

Using a list of potential maximal cliques. Suppose now that none of the steps above could be applied. In this case, we use another algorithm to solve the Minimum FILL-IN problem. This algorithm is a variant of the exact algorithm for Minimum FILL-In by Fomin and Villanger [11].

This step consists of a number of substeps:

1. We return NO if there are more than $k+1$ marked vertices.

2. We partition the unmarked vertices into groups, and return No if there are more than $3 k / 2+1$ groups.

3. We make a list of minimal separators of $G$ : this list will in general not contain all minimal separators, but it contains all that are needed to make step 5 succeed.

4. We make a list of potential maximal cliques of $G$. Again, this list does not need all potential maximal cliques, separators, but it contains all that are needed to make step 5 succeed.

5. We use the two lists above as input to an algorithm by Fomin et al. [10] and determine if there is a triangulation with fill at most $k$, and if so, find a triangulation with minimum fill.

Each of these steps, with details and correctness, will be discussed extensively below, but we start with deriving a number of graph theoretic lemmas.

From the fact that none of the above steps can be applied, several conclusions can be drawn. Let $S_{v}$ be a minimal separator contained in $N(v)$ for an unmarked vertex $v$, and let $W_{v}$ be a connected component of $G[V \backslash N[v]]$ which is full for $S_{v}$. The graph obtained by adding the two edges in $F\left(S_{v}\right)$ to $G\left[N\left[W_{v}\right]\right]$ will not be chordal, since that would imply a vertex $w \in W_{v}$, where $S_{v} \subseteq N(w)$, and thus the endpoints of an edge of $F\left(S_{v}\right)$ and vertices $v, w$ would induce a 4-cycle. Since all connected components of $G[V \backslash N[v]]$ generate nonchordal subproblems by the argument above, we can notice that $G[V \backslash N[v]]$ contains exactly one connected component, since zero components would imply that $v$ is universal, and more than one would imply that the previous described rule could be applied.

As a consequence of the above, for any unmarked vertex $v, N(v)$ contains only one minimal separator, which we will denote by $S_{v}$, and there is only one connected component in $G[V \backslash$ $N[v]]$ which is full for $S_{v}$; we will denote this connected component by $W_{v}$. Notice also that $G[N[v]]=G\left[V \backslash W_{v}\right]$ becomes chordal when the two elements of $F\left(S_{v}\right)$ are added to $G$ as fill edges. Before starting to describe the subroutine to handle this final case, we need more knowledge about the problem instance. 
Lemma 13 Given a graph $G=(V, E)$ where none of the rules above can be applied, let $u$ and $v$ be unmarked vertices, where $S_{u} \subset N[v]$. Then $u$ and $v$ are contained in the same connected component $W_{v}$ of $G\left[V \backslash S_{v}\right]$.

Proof. Let $C_{v}$ be the full component of $S_{v}$ containing $v$, and let $W_{v}$ be the full component not containing $v$. Let $C_{u}$ and $W_{u}$ be defined in the same way for $u$.

Vertices $u$ and $v$ are adjacent by definition if $v \in S_{u}$. If $v \notin S_{u}$, then both $u$ and $v$ have the endpoints of the edges in $F\left(S_{u}\right)$ in their neighborhood, and thus $u$ and $v$ are adjacent, since there are no chordless 4-cycles.

Vertex $v$ is contained in $C_{v}$ by definition, and by the edge $\{u, v\}$ vertex $u$ is contained in $N\left[C_{v}\right]=C_{v} \cup S_{v}$. If $u \in C_{v}$ then the lemma follows. Let us on the contrary assume that $u \in S_{v}$. Since $u \in S_{v}$ and $S_{v}$ is a minimal separator, then there exists a neighbour $w$ of $u$ in $W_{v}$. This implies that $W_{v} \subset C_{u}$, since $G[V \backslash N[u]]$ only contains one component $W_{u}$ and $W_{v} \cap S_{u}=\emptyset$. Since $W_{v} \subset C_{u}$, then by the previous observation that $G[V \backslash N[u]]$ only contains one component $W_{u}$ and the fact that $W_{v} \subset C_{u}$, we have that $W_{v} \cup S_{v} \subseteq N[u]$. This again implies that $S_{u}$ and $S_{v}$ are non crossing, and thus $W_{u} \subseteq N[v] \backslash S_{v}$. Now we have a contradiction by Theorem of [22], since completing $S_{v}$ into a clique makes the graph $G[N[v]]$ where the edges $F\left(S_{v}\right)$ are added chordal, while adding the edges $F\left(S_{u}\right)$ where $S_{u} \subset N[v], S_{u}, S_{v}$ are non crossing, and $W_{u} \subset N[v]$, makes $G\left[N\left[W_{u}\right]\right]$ non chordal.

Lemma 14 Given a graph $G=(V, E)$ where none of the rules above can be applied, let $u$ and $v$ be unmarked vertices. Then $N[u]=N[v]$, or $F\left(S_{u}\right) \cap F\left(S_{v}\right)=\emptyset$.

Proof. Suppose that the lemma does not hold for unmarked vertices $u$ and $v$ with corresponding minimal separators $S_{u}$ and $S_{v}$. We consider two cases. Note that $\left|F\left(S_{u}\right)\right|=2$ and $\left|F\left(S_{v}\right)\right|=2$.

In the first case, we assume that $\left|F\left(S_{u}\right) \cap F\left(S_{v}\right)\right|=1$. Let $\{x, y\}$ be the edge in $F\left(S_{u}\right) \cap$ $F\left(S_{v}\right)$. Then edge $\{u, v\}$ is contained in $E$, since the graph would otherwise contain a 4-cycle.

There are now two subcases: either $v \in S_{u}$ (equivalently $u \in S_{v}$ ) or not. If $v \notin S_{u}$, then $v$ is contained in the connected component $C_{u}$ of $G\left[V \backslash S_{u}\right]$ that contains $u$, because of the edge $\{u, v\}$. By the previous rules we know that $G\left[N\left[C_{u}\right]\right]$ is chordal when the two edges in $F\left(S_{u}\right)$ are added. Since $v$ does not have any neighbors outside of $N\left[C_{u}\right]$, and $\left|F\left(S_{v}\right)\right|=2$, then $F\left(S_{u}\right)=F\left(S_{v}\right)$, which is a contradiction to the assumption. If $v \in S_{u}$, then $S_{u} \subset N[v]$, since an edge $\{v, z\} \in F\left(S_{u}\right)$ for $z \in\{x, y\}$ would make either $x$ or $y$ non adjacent to $v$. By Lemma $13 u$ and $v$ are contained in the same connected component of $G\left[V \backslash S_{v}\right]$, and thus the arguments of the first case, where $u \notin S_{v}$, can be applied again.

In the second case, we assume that $\left|F\left(S_{u}\right) \cap F\left(S_{v}\right)\right|=2$. We will now show that $N[u]=$ $N[v]$ and thus obtain a contradiction. Like in the first half of the proof we can notice that $\{u, v\} \in E$, since otherwise a 4-cycle exists, consisting of $u, v$ and the endpoints of an edge in $F\left(S_{v}\right)$. Also there is no vertex $w$ in $S_{u} \backslash N(v)$ (equivalently $S_{v} \backslash N(u)$ ) since this would create a 4-cycle with $v, w$ (equivalently, $u, w$ ) and the endpoints of an edge of $F\left(S_{u}\right)$.

Finally we consider the case where there is a vertex $w \in\left(N(u) \backslash S_{u}\right) \backslash N(v)$. There are two subcases again, either $v \in S_{u}$ or $v \notin S_{u}$. Let us first consider the case where $v$ is not in $S_{u}$. Then $u, v, w$ are in $G[N[u]]$, which becomes chordal after adding the fill edges $F\left(S_{u}\right)$. There exists a minimal separator $S_{w}$ in $N[w] \subseteq N[u]$ that separates $w$ and $v$. Notice that $S_{w}$ becomes a clique after adding the fill edges $F\left(S_{u}\right)$ to $G[N[u]]$, since none of these fill edges 
are incident to $w$. This construction requires that $v, w$ and the endpoints of an edge in $F\left(S_{w}\right)$ induce a 4-cycle, which is a contradiction.

The second subcase is when $v \in S_{u}$. Since $F\left(S_{u}\right)=F\left(S_{v}\right)$, then none of the elements of $F\left(S_{u}\right)$ or $F\left(S_{v}\right)$ are incident to $u$ or $v$. As a result $S_{u} \subseteq N[v]$ if $v \in S_{u}$. By Lemma $13 u$ is not contained in $S_{v}$, and the arguments for the case where $u \notin S_{v}$ can be applied.

Lemma 15 Given a graph $G=(V, E)$ where none of the rules above can be applied, let $v$ be an unmarked vertex. Then the number of unmarked vertices in $S_{v}$ is at most 3.

Proof. Let us on the contrary assume that four unmarked vertices $w_{1}, w_{2}, w_{3}, w_{4}$ are contained in $S_{v}$. By Lemma $14 F\left(S_{u}\right) \cap F\left(S_{v}\right)=\emptyset$ for $u \in\left\{w_{1}, w_{2}, w_{3}, w_{4}\right\}$, since otherwise $N(u)=N(v)$ which would be a contradiction to the existence of $u \in S_{v}$. If $S_{v} \subset N[u]$ for $u \in\left\{w_{1}, w_{2}, w_{3}, w_{4}\right\}$, then by Lemma $13 N[v] \subseteq N[u]$, and since $\left|F\left(S_{v}\right)\right|=2$ and $G[N[u]]$ is triangulated by adding the two edges in $F\left(S_{u}\right)$, then $F\left(S_{u}\right)=F\left(S_{v}\right)$. This is not possible since by Lemma $14 N[u]=N[v]$ in this case. We can now conclude that $S_{v} \not \subset N[u]$ for $u \in\left\{w_{1}, w_{2}, w_{3}, w_{4}\right\}$. A consequence of this is that $F\left(S_{v}\right)$ have elements incident to all four vertices $\left\{w_{1}, w_{2}, w_{3}, w_{4}\right\}$, which is a contradiction to the fact that $\left|F\left(S_{v}\right)\right|=2$ and that these two elements have a common endpoint.

Lemma 16 Given a graph $G=(V, E)$ with no chordless 4-cycle or clique separator, let $H=(V, F)$ be a triangulation of $G$ with $E \subset F$. Then only one connected component of $(V, F \backslash E)$ contains edges.

Proof. Notice that every minimal separator of $H$ contains a fill edge, and thus every maximal clique of $H$ also contains a fill edge. Since every minimal separator of $H$ is contained in at least two maximal cliques of $H[4,13,26]$, it is enough to show that the fill edges inside any maximal clique induce a connected graph. For fill edges that belong to different maximal cliques, there is a series of minimal separators between the two cliques (in any clique tree) containing fill edges, and hence if the fill edges of each maximal clique are connected, so are the fill edges of the whole graph. Let $X$ be a maximal clique of $H$, and assume that $\{u, v\}$ and $\{x, y\}$ are fill edges in $X$. There are two cases; either one of the edges $\{x, u\},\{u, y\},\{y, v\},\{v, x\}$ is a fill edge and the lemma holds, or $\{x, u\},\{u, y\},\{y, v\},\{v, x\}$ are edges of $G$ that induce a 4-cycle which is a contradiction.

A consequence of this lemma is that there are at most $k+1$ vertices incident to a fill edge. Hence the first substep: we return NO at this point if there are more than $k+1$ marked vertices. So assume there are at most $k+1$ marked vertices.

The next step of the algorithm is to control the unmarked vertices. What we will do is to partition the unmarked vertices into groups, bound the number of groups, and show that an algorithm by Fomin et. al. [10] has a running time that can be bounded by a function of the number of groups and marked vertices.

For each unmarked vertex $v$ we know that $\left|F\left(S_{v}\right)\right|=2$, and by Lemma 14, we know that for pairs of unmarked vertices $u$ and $v, F\left(S_{v}\right)=F\left(S_{u}\right)$, or $F\left(S_{v}\right) \cap F\left(S_{u}\right)=\emptyset$. Partition the unmarked vertices into groups, where $F\left(S_{v}\right)=F\left(S_{u}\right)$ for all pairs $u, v$ of vertices in the same group.

Lemma 17 If $G$ has a triangulation with at most $k$ fill edges, then there are at most $3 k / 2+1$ groups of unmarked vertices. 
Proof. Consider a minimum triangulation $H$, which respects all given marks. By Lemma 16 there are at most $k+1$ vertices incident to fill edges of $H$. Since $N[u]=N[v]$ for a pair of unmarked vertices in the same group, we can notice that either all or none of them will have an incident fill edge in $H$. There are at most $k+1$ groups that have fill edges incident to its vertices. The number of groups that do not have fill edges incident to its vertices is at most $k / 2$, since each such group has two private fill edges in its neighborhood.

So, if we have more than $3 k / 2+1$ groups, we return NO.

The final step of our algorithm is to apply an algorithm by Fomin et al. [10] to compute a minimum triangulation, with one important modification concerning the groups of unmarked vertices. Fomin et al. [10] have shown that a minimum triangulation can be computed in $\mathcal{O}\left(\left(\left|\Delta_{G}\right|+\left|\Pi_{G}\right|\right) \cdot n^{3}\right)$ time, where $\Delta_{G}$ is the set of minimal separators of $G$, and $\Pi_{G}$ is the set of potential maximal cliques of $G$. The algorithm of [10] is more powerful than stated. Inspection of this algorithm shows that it also can be used to obtain the following result.

Theorem 18 (See Fomin et al. [10]) There is an algorithm that, given a graph $G=(V, E)$, a list $L_{1}$ of potential maximal cliques in $G$, and a list $L_{2}$ of minimal separators in $G$, returns in time $\mathcal{O}\left(n^{3}\left(\left|L_{1}\right|+\left|L_{2}\right|\right)\right)$ a triangulation $H$ of $G$, such that

- each maximal clique in $H$ is an element of $L_{1}$

- each minimal separator in $H$ is an element of $L_{2}$

- the fill of $H$ is minimal amongst all triangulations that fulfil the above two criteria.

We can use this result as follows: we list potential maximal cliques and minimal separators of $G$, but we can avoid listing such potential maximal cliques and minimal separators when they would imply more than $k$ fill edges. As minimal separators in a chordal graph are cliques, we only have to list minimal separators $S$ with $|F(S)| \leq k$ and potential maximal cliques $\Omega$ with $|F(\Omega)| \leq k$, when we want to solve the Minimum FILL-IN problem with parameter $k$.

It is not hard to see that if $u$ and $v$ have the same closed neighborhood, then each potential maximal clique that contains $u$ also contains $v$; the same holds for each minimal separator in a minimal triangulation of $G$. A vertex is for instance contained in a minimal $u, v$-separator $S$ if and only if it has a neighbor in the connected components of $G[V \backslash S]$ which contains respectively $u$ and $v$. For potential maximal cliques there exists a similar definition. A vertex is contained in the potential maximal clique if and only if it can reach all other vertices in the potential maximal clique by direct edges or paths consisting only of vertices that are not contained in the potential maximal clique. Given these arguments, no vertex set that contains only a part of a group of unmarked vertices are candidates to be minimal separators or potential maximal cliques. Thus, when listing minimal separators and potential maximal cliques, we can treat vertices with the same closed neighborhood as a single vertex.

By Lemma 15, for each group of unmarked vertices, there are at most three unmarked vertices not in this group that are incident to some vertices in the group. Consider a collection of $4 \sqrt{k}$ of the $3 k / 2+1$ groups of unmarked vertices. The set of vertices in these groups can never belong to a clique in a triangulation with at most $k$ fill edges, as there are more than $k$ pairs of vertices in this set that are non-adjacent. Thus, we can limit our lists of minimal separators and potential maximal cliques to sets that contain at most $4 \sqrt{k}$ of the $3 k / 2+1$ groups of unmarked vertices. 
Lemma 19 Given a graph $G$, let $M$ be the set of marked vertices, let $q$ be the number of groups of unmarked vertices, and let $k \leq|M|-1$. Then all minimal separators and potential maximal cliques containing at most $\ell$ groups of unmarked vertices can be listed in $\mathcal{O}^{*}\left(1.7549^{k+1} \cdot \sum_{i=0}^{\ell}\left(\begin{array}{l}q \\ i\end{array}\right)\right)$ time and space.

Proof. To prove Lemma 19, we show that all minimal separators and potential maximal cliques containing $W \subset(V \backslash M)$ where $|W| \leq \ell$ can be listed in $\mathcal{O}^{*}\left(1.7549^{k+1}\right)$ time and space. For the rest of this proof we assume $W$ is the intersection between $V \backslash M$ and the minimal separator or potential maximal clique we are searching for.

Due to Proposition 1 every minimal separator $S$ has two full components $C_{1}$ and $C_{2}$. Without loss of generality let $C_{1}$ be the component such that $\left|C_{1} \cap M\right| \leq\left|C_{2} \cap M\right|$. Fomin and Villanger [11] give an algorithm that lists all minimal separators by searching for $C_{1}$, starting from every vertex of $G$. Since the inclusion/exclusion of unmarked vertices is already decided, we only have to branch on marked vertices, and thus the algorithm of [11] can be adapted to list all the minimal separators containing $W$ and not $V \backslash(M \cup W)$ in $\mathcal{O}^{*}\left(1.6181^{k+1}\right)$ time and space.

Listing potential maximal cliques is done in the same way, but is slightly more involved. The set of potential maximal cliques can be partitioned into two sets, nice potential maximal cliques and non nice potential maximal cliques. A potential maximal clique is defined as nice if it is not an induced clique when all but one minimal separator completely contained in the potential maximal clique is completed into a clique. By $[3,10,11]$ the set of non nice potential maximal cliques containing at most $\ell$ unmarked vertices can be generated from the set of minimal separators and nice potential maximal cliques contains at most $\ell$ unmarked vertices with a polynomial delay for each potential maximal clique. Our problem is then reduced to listing nice potential maximal cliques where the graph induced over these contains at most $\ell$ unmarked vertices.

One property for a nice potential maximal clique $\Omega$ is the existence of a connected vertex set $Z$ and a vertex $x \notin \Omega$ such that $\Omega=N(Z) \cup\{x\}$, and $Z \cap \Omega=\emptyset$ and $Z$ contains at most $2 / 3$ of the vertices not in $\Omega[25]$.

Again we use the algorithm in [11] and search for the connected vertex set $Z$. In the same way as for minimal separators we do not have to branch on unmarked vertices $(V \backslash M)$. As a result, the algorithm for listing potential maximal cliques in [11] can be adapted to list all nice potential maximal cliques containing the unmarked vertices $W$ in $\mathcal{O}^{*}\left(1.7549^{k+1}\right)$ time and space. Given the set of minimal separators and nice potential maximal cliques containing at most $\ell$ unmarked vertices the set of non nice potential maximal cliques containing at most $\ell$ unmarked vertices can be generated with a polynomial delay [11].

As discussed above, minimal separators and potential maximal cliques that contain vertices from more than $4 \sqrt{k}$ groups cannot belong to a triangulation with at most $k$ fill edges. Hence, we apply Lemma 19 with $\ell=4 \sqrt{k}$. Thus, in $\mathcal{O}^{*}\left(1.7549^{k}\right)$ time, we list all minimal separators and potential maximal cliques that can contribute to a triangulation with at most $k$ fill edges. Given these lists, we now employ the algorithm of Fomin et al. [10] (see Theorem 18) and compute a triangulation with minimum fill of $G$; this algorithm uses time, polynomial in $n$ times the size of the list of potential maximal cliques.

Note that the algorithm in Theorem 18 does not need to respect the marks. Thus, we possibly obtain for the subproblem a solution while there is no solution for this subproblem 
respecting the mark; as discussed at the start of this section, this does not harm the overall correctness of the algorithm.

We obtain the following condition for the running time: $T(k, r) \leq 1.7549^{k}$, and thus $a \geq 1.7549$.

Analyzing the running time. We derived the following conditions on $a$ and $b$, such that, if these hold, then by induction it follows that $T(k, r) \leq a^{k} b^{k} \cdot o(k)$ : for all integers $\alpha \geq 2$ : $a^{3 \alpha-3} \geq \alpha, a \geq b, a b \geq 2, a^{3} b^{2} \geq 1+a^{3} b, a^{6} b \geq 2+a^{6}, a \geq 1.7549$. As we start with an instance with $k$ and $r=2 k$, the running time is a polynomial in $n$ times $T(k, 2 k)$. We get as minimum $a b^{2}=2.35965$ when we set $a=1.7549$ and $b=1.15956$. Rounding this up allows to ignore the $o(k)$ term, and thus the algorithm requires $\mathcal{O}^{*}\left(2.35965^{k}\right)$ time. By the same arguments as the ones used for the polynomial part of the running time of our previous algorithm, we can conclude that this algorithm has running time $\mathcal{O}\left(k^{2} n m+2.35965^{k}\right)$, and requires $\mathcal{O}^{*}\left(1.7549^{k}\right)$ space.

Theorem 20 The Minimum FiLL-In problem can be solved in $\mathcal{O}\left(k^{2} n m+2.35965^{k}\right)$ time, using $\mathcal{O}^{*}\left(1.7549^{k}\right)$ space.

\section{Conclusions}

In this paper, we presented parameterized algorithms for the Minimum FILL-In problem. The first algorithm is relatively simple and uses polynomial space; the second algorithm uses for one step exponential space, but less time as a function of $k$. We expect that the first algorithm is practical for small variants of $k$. Using some of the steps of the second algorithm in combination with the first algorithm probably gives speedup for many inputs. It would be an interesting study to experimentally evaluate the first algorithm, and variants of it where some of the steps of the second algorithm are added to it.

The bounding conditions in the analysis of the running time of the second algorithm are $a \geq 1.7549$, and $a^{3} b^{2} \geq 1+a^{3} b$. Thus, a faster algorithm could be possibly obtained by either finding a faster version of the algorithm that lists the minimal separators and potential maximal cliques (i.e., speeding up the algorithm from Fomin and Villanger [11], see Lemma 19), or the two steps that give the condition $a^{3} b^{2} \geq 1+a^{3} b$ : minimal separators $S$ with $|F(S)|=3$ and the first case where we split into two non-chordal graphs.

We obtain running times of the form $O\left(k^{2} n m+c^{k}\right)$ time by first applying the kernelization algorithm for Minimum Fill-In by Kaplan et al. [17], see also [20]. To obtain an algorithm with a better asymptotic running time, it would be useful to have an algorithm that obtains a kernel of size polynomial in $k$ for Minimum FilL-In whose running time is $o\left(k^{2} n m\right)$.

\section{Acknowledgements}

We thank Guido Diepen, Igor Razgon, Thomas van Dijk, and Johan van Rooij for useful discussions. 


\section{References}

[1] A. Berry And J. Bordat, Moplex elimination orderings, Electronic notes in Discrete Mathematics, 8 (2001), pp. 6-9.

[2] A. Berry, P. Heggernes, and Y. Villanger, A vertex incremental approach for maintaining chordality, Discrete Mathematics, 306 (2006), pp. 318-336.

[3] V. Bouchitté And I. Todinca, Listing all potential maximal cliques of a graph., Theoretical Computer Science, 276 (2002), pp. 17-32.

[4] P. Buneman, A characterization of rigid circuit graphs, Discrete Mathematics, 9 (1974), pp. $205-212$.

[5] L. CAI, Fixed-parameter tractability of graph modification problems for hereditary properties, Information Processing Letters, 58 (1996), pp. 171-176.

[6] F. R. K. Chung And D. Mumford, Chordal completions of planar graphs, Journal of Combinatorial Theory, Series B, 31 (1994), pp. 96-106.

[7] G. A. DiRAc, On rigid circuit graphs, Anh. Math. Sem. Univ. Hamburg, 25 (1961), pp. $71-76$.

[8] R. G. Downey and M. R. Fellows, Parameterized complexity, Springer-Verlag, New York, 1999.

[9] J. Flum And M. Grohe, Parameterized Complexity Theory, Springer-Verlag, New York, 2006 .

[10] F. V. Fomin, D. Kratsch, And I. TodincA, Exact (exponential) algorithms for treewidth and minimum fill-in, in Proceedings of the 31st International Colloquium on Automata, Languages and Programming, ICALP 2004, vol. 3142 of Lecture Notes in Computer Science, Springer, 2004, pp. 568-580.

[11] F. V. Fomin And Y. Villanger, Treewidth computation and extremal combinatorics, in Proceedings of the 35th International Colloquium on Automata, Languages and Programming, ICALP 2008, vol. 5125 of Lecture Notes in Computer Science, Springer, 2008, pp. $210-221$.

[12] D. R. Fulkerson And O. A. Gross, Incidence matrices and interval graphs, Pacific Journal of Mathematics, 15 (1965), pp. 835-855.

[13] F. GAVRIL, The intersection graphs of subtrees in trees are exactly the chordal graphs, Journal of Combinatorial Theory, Series B, 16 (1974), pp. 47-56.

[14] M. C. Golumbic, Algorithmic Graph Theory and Perfect Graphs, Academic Press, New York, 1980.

[15] P. Heggernes, Minimal triangulations of graphs: A survey, Discrete Mathematics, 306 (2006), pp. 297-317. 
[16] H. Kaplan, R. Shamir, and R. E. Tarjan, Tractability of parameterized completion problems on chordal and interval graphs: Minimum fill-in and physical mapping, in Proceedings of the 35th annual symposium on Foundations of Computer Science, FOCS'94, IEEE Computer Science Press, 1994, pp. 780-791.

[17] — Tractability of parameterized completion problems on chordal, strongly chordal, and proper interval graphs, SIAM Journal on Computing, 28 (1999), pp. 1906-1922.

[18] S. L. LAuritzen AND D. J. Spiegelhalter, Local computations with probabilities on graphical structures and their applications to expert systems, Journal of the Royal Statistical Society, Series B, 50 (1988), pp. 157-224.

[19] C. LeKKerkerker and J. Boland, Representation of a finite graph by a set of intervals on the real line, Fund. Math., 51 (1962), pp. 45-64.

[20] A. Natanzon, R. Shamir, and R. Sharan, A polynomial approximation algorithm for the minimum fill-in problem, SIAM Journal on Computing, 30 (2000), pp. 1067-1079.

[21] R. Niedermeier, Invitation to Fixed-Parameter Algorithms, Oxford University Press, 2006 .

[22] A. PARRA AND P. SChefFler, Characterizations and algorithmic applications of chordal graph embeddings, Discrete Applied Mathematics, 79 (1997) pp. 171-188.

[23] D. J. Rose, Triangulated graphs and the elimination process, Journal of Mathematical Analysis and Applications, 32 (1970), pp. 597-609.

[24] R. E. Tarjan and M. Yannakakis, Simple linear-time algorithms to test chordality of graphs, test acyclicity of hypergraphs, and selectively reduce acyclic hypergraphs, SIAM Journal on Computing, 13 (1984), pp. 566-579.

[25] Y. Villanger, Improved exponential-time algorithms for treewidth and minimum fill-in., in Proceedings of the 7th Latin American Theoretical Informatics Symposium, LATIN 2006, vol. 3887 of Lecture Notes in Computer Science, Springer, 2006, pp. 800-811.

[26] J. Walter, Representations of rigid cycle graphs, PhD thesis, Wayne State University, USA, 1972.

[27] M. Yannakakis, Computing the minimum fill-in is NP-complete, SIAM Journal on Algebraic and Discrete Methods, 2 (1981), pp. 77-79. 